\title{
Paeoniflorin inhibits proliferation and promotes autophagy and apoptosis of sweat gland cells
}

\author{
YUAN XU ${ }^{1,2^{*}}, \mathrm{HONG} \mathrm{HE}^{3}, \mathrm{PING} \mathrm{LI}^{2^{*}}$ and HONGWEI LIU ${ }^{1}$ \\ ${ }^{1}$ Department of Plastic Surgery, The First Affiliated Hospital of Jinan University, Guangzhou, Guangdong 510630; \\ ${ }^{2}$ Department of Plastic Surgery, The First Affiliated Hospital of Guangdong Pharmaceutical University; \\ ${ }^{3}$ Health Care and Physical Examination Center, The First Affliated Hospital, Sun \\ Yat-sen University, Guangzhou, Guangdong 510080, P.R. China
}

Received May 27, 2021; Accepted September 8, 2021

DOI: $10.3892 /$ etm.2021.10975

\begin{abstract}
Axillary bromhidrosis is sweat excreted by apocrine glands in the armpits, mouth corners and other parts. The clinical manifestation includes excessive sweating and heavy odor, leading to the growth of bacteria and skin disease. The present study investigated the mechanism underlying the effect of paeoniflorin (PF) in the treatment of bromhidrosis. PF was injected into the feet of rats, and the foot skin was dissected for histological analysis. Primary human sweat gland cells (hSGCs) were isolated from patients with bromhidrosis. After $24 \mathrm{~h}$ treatment with PF or 3-methyladenine, the production of reactive oxygen species (ROS), autophagy, apoptosis, proliferation and cell cycle distribution were determined. PF induced nuclear pyknosis in rat SGCs. In vitro PF treatment inhibited cell proliferation with a $25 \%$ inhibitory concentration of $9.530 \mu \mathrm{M}$. Treatment with $9.530 \mu \mathrm{M}$ PF for $24 \mathrm{~h}$ significantly increased apoptosis, ROS production and autophagy in hSGCs. PF promoted LC3B and Beclin 1 expression, but inhibited p62, phosphorylated (p)-PI3K and p-Akt expression. 3-methyladenine treatment reversed $\mathrm{PF}$-induced changes in hSGCs. PF-induced inhibition of hSGC proliferation was associated with ROS production, apoptosis, and autophagy. These findings provide a basis for treating bromhidrosis.
\end{abstract}

Correspondence to: Dr Hongwei Liu, Department of Plastic Surgery, The First Affiliated Hospital of Jinan University, 613 West Huangpu Avenue, Guangzhou, Guangdong 510630, P.R. China E-mail: liuhongwei0521@hotmail.com

*Contributed equally

Abbreviations: 3-MA, 3-methyladenine; hSGCs, human sweat gland cells; IC25, 25\% inhibitory concentration; IC50, 50\% inhibitory concentration; PF, paeoniflorin; SMCs, smooth muscle cells; CK8, cytokeratin 8; SMA, $\alpha$-smooth muscle actin

Key words: autophagy, axillary bromhidrosis, hSGCs, proliferation

\section{Introduction}

Axillary bromhidrosis is a common dermatological condition among the general population, and its overall worldwide prevalence is reported to be $1-3 \%(1,2)$. Bromhidrosis has a negative effect on a person's quality of life (3). The treatments for bromhidrosis include surgery, physical therapy and botulinum toxin A injection (4,5).

Bromhidrosis results from hyperactivity of axillary sweat gland (SG)s, and most treatment methods focus on removing the axillary $S G$ and decreasing axillary sweating $(3,6)$. However, there is no radical cure for bromhidrosis and the development of novel drugs or therapies for managing bromhidrosis remains important. The etiology of bromhidrosis is multifactorial (7) and is associated with infection (8) as well as metabolic and neurological dysfunction (9). However, the exact etiology of bromhidrosis is unclear.

Paeoniflorin (PF), an agent isolated from Paeoniae alba, is the primary active component in Shaobei injection (10). PF has been shown to promote production of intracellular $\mathrm{Ca}^{2+}$ in salivary gland cells (11) and exert a neuroprotective effect (12) by inducing autophagy-associated pathways (13). In addition, PF regulates the metabolism of amino acids, cholesterol and fat (14-16) and inhibits proliferation of fibroblast-like synoviocytes (17) and pulmonary artery smooth muscle cells (SMCs) (18). To the best of our knowledge, however, the effects of PF on SG cells (SGCs) and its mechanism in treating bromhidrosis have not been reported.

In clinical practice, patients with axillary bromhidrosis who had been injected with Shaobei under their armpits reported that axillary bromhidrosis was notably decreased. Therefore, the present study aimed to determine the effect of PF on bromhidrosis both in vivo and in vitro. The effect of PF on SG morphology, as well as proliferation, apoptosis and autophagy of SGCs was determined.

\section{Materials and methods}

Animals and treatment. The protocols for all animal experiments were approved by the Institutional Animal Care and Use Committee of the First Affiliated Hospital of Guangdong 
Pharmaceutical University. Male Sprague-Dawley rats $(\mathrm{n}=25$; age, 3-4 weeks; weight, $210 \pm 15 \mathrm{~g}$ ) were purchased from the Experimental Animal Center of Sun Yat-sen University and housed at $27^{\circ} \mathrm{C}$, relative humidity $45 \%$, light/dark cycle of $12 \mathrm{~h}$. Rat chow and water were available ad libitum. PF $(2.5 \mathrm{mg} / \mathrm{kg}$ body weight $)$ was injected into the upper right claw via foot injection. At $6,24,48$ and $72 \mathrm{~h}$ post-treatment ( $\mathrm{n}=5 /$ group), animals were sacrificed by overdose of pentobarbital sodium ( $135 \mathrm{mg} / \mathrm{kg}$; R\&D Systems, Inc.). The foot skin was dissected and prepared for histological examination. All experiments were performed in accordance with the Chinese regulations on the use and breeding of experimental animals (19).

Histological examination. The foot skin samples were cut into pieces, fixed with $4 \%$ paraformaldehyde at room temperature for 48 h (cat. no. p1110; Beijing Solarbio Science \& Technology Co., Ltd.), then dehydrated, made transparent, and embedded in paraffin. Next, the embedded tissues were cut into serial sections (5 $\mu \mathrm{m}$ thickness) using a microtome. After baking the sections of tissue at $60^{\circ} \mathrm{C}$ for $2 \mathrm{~h}$, they were immersed in xylene, ethanol $(100,95,80$ and $70 \%)$, and pure water, counterstained with hematoxylin-eosin at $37^{\circ} \mathrm{C}$ for $10 \mathrm{~min}$, dehydrated with gradient ethanol and mounted with neutral resin as previously reported (20). The photos were captures at the magnifications of $x 20$ and $\times 400$ by an Olympus light microscope (cat. no. BX51; Olympus Corporation) and the data were analyzed using Olympus Stream software (Olympus Corporation).

Cell isolation and culture conditions. Primary human SGCs (hSGCs) were isolated from the alar skin of five patients with bromhidrosis (three men and two women; age, 25-45 years) recruited between June and September 2020 at The First Affiliated Hospital of Guangdong Pharmaceutical University (Guangzhou, China). Bromhidrosis was diagnosed according to previously reported criteria (21). The protocols for all experiments involving humans were approved by the Institutional Review Board and Ethics Committee of The First Affiliated Hospital of Guangdong Pharmaceutical University Written (approval no. 202182). Written informed consent was obtained from each of the five patients prior to enrollment in the study. Primary hSGCs were isolated as previously reported (22). The pieces of foot skin were cleaned of subcutaneous fat, washed with precooled PBS, then incubated with dispase $(0.8 \mathrm{U} / \mathrm{ml}$; Roche Diagnostic Ltd.) at $37^{\circ} \mathrm{C}$ for $16-20 \mathrm{~h}$. The dermis was obtained and subsequently digested with collagenase type IV (2.5 mg/ml; Gibco; Thermo Fisher Scientific, Inc.) at $37^{\circ} \mathrm{C}$ for $1 \mathrm{~h}$. The hSGCs were then carefully collected from the lysate using a Transferpettor under a microscope. Next, the hSGCs were cultured in DMEM-F12 (Gibco; Thermo Fisher Scientific, Inc.) supplemented with FBS, $1 \%$ penicillin/streptomycin (Beyotime Institute of Biotechnology), 10X Insulin Transferrin Selenium, 2 mM l-glutamine (Gibco; Thermo Fisher Scientific, Inc.), $2 \mathrm{nmol} / \mathrm{ml}$ triiodothyronine (Sigma-Aldrich; Merck KGaA), $10 \mathrm{ng} / \mathrm{ml}$ recombinant human epidermal growth factor (Invitrogen; Thermo Fisher Scientific, Inc.) and $0.4 \mathrm{mg} / \mathrm{ml}$ hemisuccinate hydrocortisone (Sigma-Aldrich; Merck KGaA) at $37^{\circ} \mathrm{C}$ and $5 \% \mathrm{CO}_{2}$. Cells at passage 3-4 were used for further experiments.
Immunofluorescence assay. Isolated hSGCs were identified using cytokeratin 8 (CK8) and $\alpha$-smooth muscle actin (SMA) immunofluorescence assays. Primary cells were harvested, resuspended in DMEM-F12 and then placed $2 \times 10^{5}$ cells into 24-well plates with slides (14 $\mathrm{mm}$ in diameter; Costar; Corning, Inc.) at $37^{\circ} \mathrm{C}$ for $24 \mathrm{~h}$. Next, cells attached to slides were fixed with $4 \%$ Paraformaldehyde at room temperature for 30 min, treated with $0.5 \%$ Triton X-100 (Beyotime Institute of Biotechnology) at room temperature for $5 \mathrm{~min}$, then incubated with antibodies against CK8 (1:100; cat. no. ab53280; Abcam) and $\alpha$-SMA (1:200; cat. no. ab32535; Abcam) at $4^{\circ} \mathrm{C}$ overnight. This was followed by incubation with secondary goat anti-mouse/rabbit IgG antibody labeled with Alexa Fluor 594/488 (both 1:200; cat. no. ab150113; Abcam) at $37^{\circ} \mathrm{C}$ for $1 \mathrm{~h}$. Cell nuclei were stained with DAPI $(10 \mu \mathrm{M})$ at $37^{\circ} \mathrm{C}$ for $10 \mathrm{~min}$. Images of the stained cells were captured using a fluorescence microscope (magnification, $\mathrm{x} 20$ and $\mathrm{x} 400$; Lionheart LX; BioTek Instruments Inc.).

hSGC treatment. Following identification and passaging, hSGCs at passage 3-4 were treated with $0,2,4,8,16$, 32 and $64 \mu \mathrm{M}$ PF (Sigma-Aldrich; Merck KGaA) at $37^{\circ} \mathrm{C}$ in a $5 \% \mathrm{CO}_{2}$ atmosphere for $24 \mathrm{~h}$. For inhibition of autophagy, $\mathrm{PF}$ and/or $5 \mathrm{mM}$ 3-methyladenine (3-MA; Sigma-Aldrich; Merck $\mathrm{KGaA}$ ) were added to hSGCs at $37^{\circ} \mathrm{C}$ for $24 \mathrm{~h}$. Each condition was replicated three times.

Cell proliferation analysis. The inhibitory effect of PF on hSGC proliferation was examined using Cell Counting Kit-8 (CCK-8) assay $(5 \mathrm{mg} / \mathrm{ml}$; Beyotime Institute of Biotechnology) for $2 \mathrm{~h}$ according to the manufacturer's instructions. The absorbance of each well was read at $450 \mathrm{~nm}$ using a microplate reader (BioTek Instruments, Inc.). The 50 and 25 inhibitory concentration (IC) were calculated based on CCK-8 assay results. Untreated hSGCs were used as the control.

Measurement of intracellular reactive oxygen species (ROS) levels. Intracellular ROS levels were determined using a CellROX ${ }^{\circledR}$ Green assay kit (Invitrogen; Thermo Fisher Scientific, Inc.) Following treatment with PF, hSGCs were incubated with CellROX ${ }^{\circledast}$ probe at $37^{\circ} \mathrm{C}$ and $5 \% \mathrm{CO}_{2}$ for $30 \mathrm{~min}$, followed by three washes with DMEM (Gibco; Thermo Fisher Scientific, Inc.) without FBS. The fluorescence intensity of each group of cells was measured using a BD FACS Calibur ${ }^{\mathrm{TH}}$ flow cytometer (BD Biosciences) and analyzed by FlowJo software v10.0. N-Acetyl-Cysteine (1 $\mathrm{mM})$ and tert-butylhydroperoxide $(200 \mu \mathrm{M}$; BD Biosciences) were used as negative and positive controls, respectively.

Cell apoptosis. Cell apoptosis was determined by flow cytometry and Hoechst 33258 staining. In brief, hSGCs $\left(2 \times 10^{5}\right.$ cells $\left./ \mathrm{ml}\right)$ were placed into 24 -well plates (Costar; Corning, Inc.) and incubated with PF $(9.53 \mu \mathrm{M})$ for $24 \mathrm{~h}$ at $37^{\circ} \mathrm{C}$ in a $5 \% \mathrm{CO}_{2}$. The cells were then harvested by Trypsin, fixed with $70 \%$ ethanol at room temperature for 30 min and stained using Hoechst 33258 DNA intercalating dye (Beyotime Institute of Biotechnology) or Annexin V-FITC/PI fluorescent double staining solutions (Beyotime Institute of 

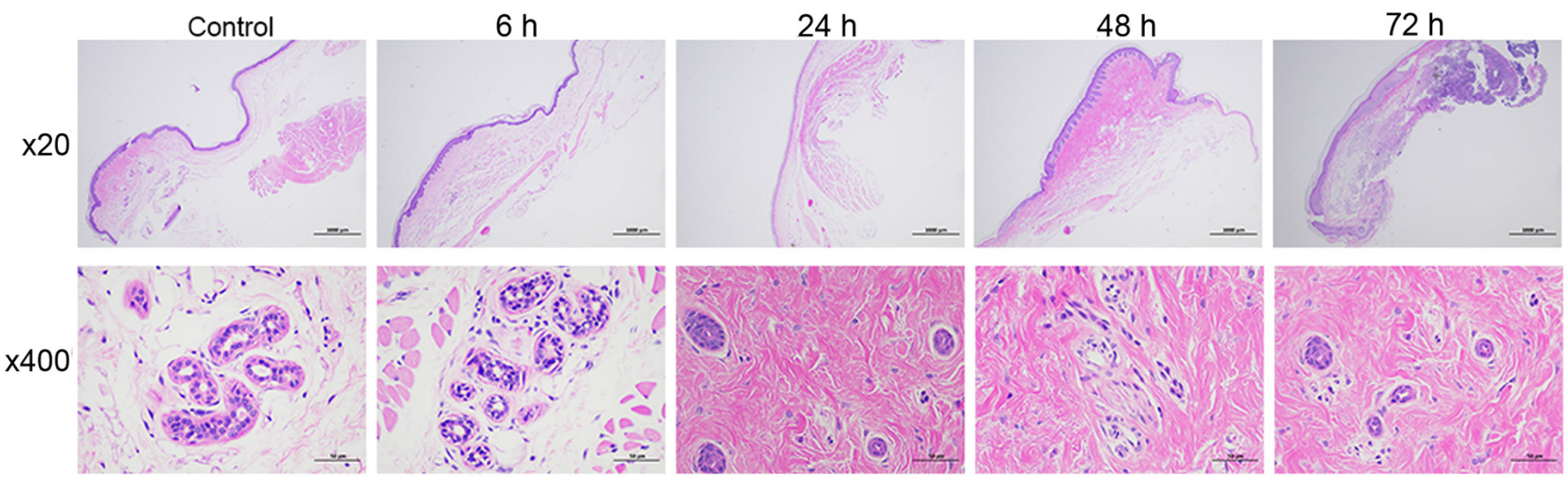

Figure 1. Histology of skin and sweat glands. Rats were treated with paeoniflorin for up to $72 \mathrm{~h}$. Magnification, x20 and x400.

Biotechnology) according to the manufacturer's instructions. An Olympus fluorescent microscope (magnification, x400) was used to record images of the Hoechst 33258-stained cells. Cell apoptosis, as indicated by Annexin V/PI fluorescent double staining, was analyzed using a FACS Calibur ${ }^{\mathrm{TM}}$ flow cytometer (BD Biosciences) and the data were analyzed using FlowJo 10.07 software (FlowJo LLC).

Cell cycle distribution analysis. The effect of PF on hSGC cell cycle distribution was analyzed using flow cytometry (BD Biosciences). hSGCs were treated with $\mathrm{PF}(9.530 \mu \mathrm{M})$ at $37^{\circ} \mathrm{C}$ for $24 \mathrm{~h}$, and then the cells were incubated with Trypsin and $4 \mathrm{~mL}$ of blocking reagent DMEM-F12 (Gibco; Thermo Fisher Scientific, Inc.) was added at $37^{\circ} \mathrm{C}$ for $10 \mathrm{sec}$. The cells washed using PBS and fixed with $70 \%$ ethanol at $4^{\circ} \mathrm{C}$ for $30 \mathrm{~min}$ prior to $50 \mu \mathrm{g} / \mathrm{ml}$ PI/RNase staining solution (Sungene Biotech Co., Ltd.) at room temperature for $20 \mathrm{~min}$. A FACS Calibur ${ }^{\mathrm{TM}}$ flow cytometer was used for cell cycle analysis and analyzed by FlowJo software v10.0.

Western blot analysis. The total cellular proteins were extracted from PF-treated and control hSGCs using lysis buffer (Beyotime Institute of Biotechnology) and protein determination by BCA Protein Assay kit (Pierce; Thermo Fisher Scientific, Inc.). Following protein quantification, an aliquot of total protein $(30 \mu \mathrm{g})$ from each extract was separated by 10\% SDS-PAGE (Invitrogen; Thermo Fisher Scientific, Inc.) and the protein bands were electro-transferred onto PVDF membranes (MilliporeSigma). Then, 5\% skimmed milk (Beyotime Institute of Biotechnology) was used to block the membrane at room temperature for $30 \mathrm{~min}$, followed by primary antibody incubation at $4{ }^{\circ} \mathrm{C}$ overnight. After washing with TBS- $0.05 \%$ Tween-20 (Sigma-Aldrich; Merck KGaA) and polysorbate buffer (Invitrogen; Thermo Fisher Scientific, Inc.), the membrane was incubated with the secondary HRP Goat anti-Rabbit IgG (1: 20000; BOSTER, cat. no. BA1054) at room temperature for $1 \mathrm{~h}$. Next, the membranes were incubated with anti-LC3B (1:1,000, cat. no. ab192890, Abcam), anti-Beclin 1 (1:1,500, cat. no. ab210498, Abcam), anti-P62 (1:1,000, cat. no. ab109012, Abcam), phosphorylated (p)-PI3K (1:1,000, cat. no. ab182651, Abcam), PI3K (1:2,000, cat. no. ab140307, Abcam), Akt (1:500, cat. no. ab8805,
Abcam), p-Akt (1:1,500, cat. no. ab38449, Abcam), and GAPDH (1:1,000, cat. no. ab8245, Abcam) primary antibodies at $4^{\circ} \mathrm{C}$ overnight. GAPDH served as a control protein. The immunostained protein bands were visualized using an enhanced chemiluminescence kit (Beyotime Institute of Biotechnology) according to the manufacturer's protocol. The optical density was analyzed by Image-Pro Plus 6.0 (Easybio Technology Co., Ltd.).

Statistical analysis. All data were analyzed using GraphPad Prism 6.0 software (GraphPad Software, Inc.) and results are expressed as the mean \pm standard deviation $(n=3)$. One way analysis of variance followed by post hoc Tukey's test was performed to compare multiple groups. $\mathrm{P}<0.05$ was considered to indicate a statistically significant difference.

\section{Results}

PF alters the histology of skin and SGs. Treatment with PF for 24, 48 or $72 \mathrm{~h}$ significantly induced nuclear pyknosis in SGs (Fig. 1). At 48 h, SGCs were invisible and small numbers of inflammatory cells were present. In addition, the cytoplasm of glandular epithelial cells from rats treated with $\mathrm{PF}$ appeared to be loose compared with cytoplasm in control cells. Treatment with PF for a short period of time $(6 \mathrm{~h})$ did not change the histology of skin and SGs.

PF inhibits proliferation of $h S G C s$. CK8 and $\alpha$-SMA are specific markers of SGCs $(23,24)$. To identify SGCs, localization was confirmed by immunohistological analysis of these maker proteins. Fluorescent images of cells treated with CK8 and $\alpha$-SMA showed that hSGCs had been successfully isolated from human alar skin (Fig. 2A). Primary hSGCs were treated with a series of PF concentrations; PF inhibited hSGC proliferation in a dose-dependent manner (Fig. 2B). The $\mathrm{IC}_{50}$ and $\mathrm{IC}_{25}$ values for PF were 41.013 and $9.530 \mu \mathrm{M}$, respectively. After considering the toxicity of PF to human skin (25), 9.530 $\mu \mathrm{M}$ PF was subsequently selected for use in treating hSGCs.

PF promotes intracellular ROS production and apoptosis. Treatment with $9.530 \mu \mathrm{M} \mathrm{PF}$ for $24 \mathrm{~h}$ 


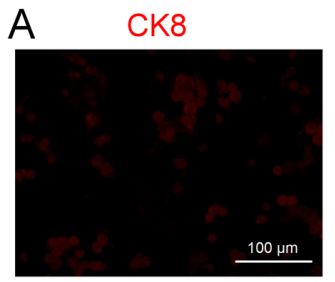

a-SMA

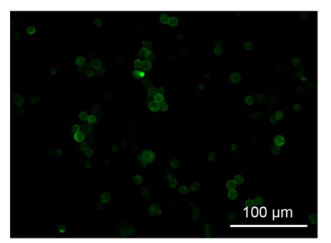

DAPI

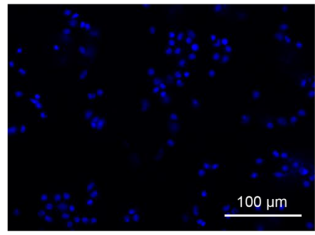

DAPI

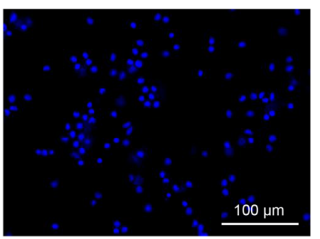

CK8/DAPI

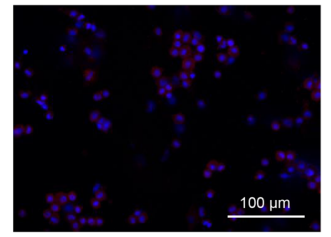

a-SMA/DAPI

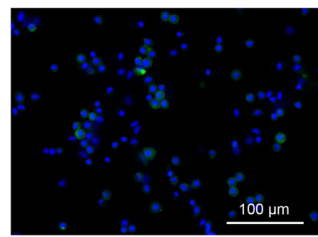

B

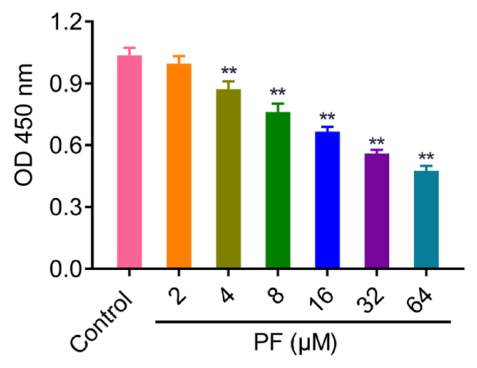

Figure 2. Identification of hSGCs and response to PF. (A) Primary hSGCs expressing CK8 and $\alpha$-SMA. (B) Proliferation of hSGCs treated with PF for $24 \mathrm{~h}$. ${ }^{* *} \mathrm{P}<0.01$ vs. control. PF, paeoniflorin; hSGC, human sweat gland cell; CK8, cytokeratin 8 ; $\alpha$-SMA, $\alpha$-smooth muscle actin; OD, optical density.

A 01

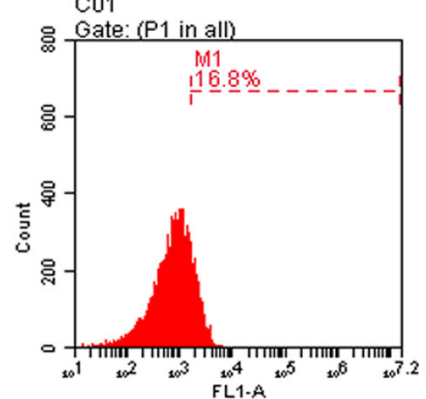

B
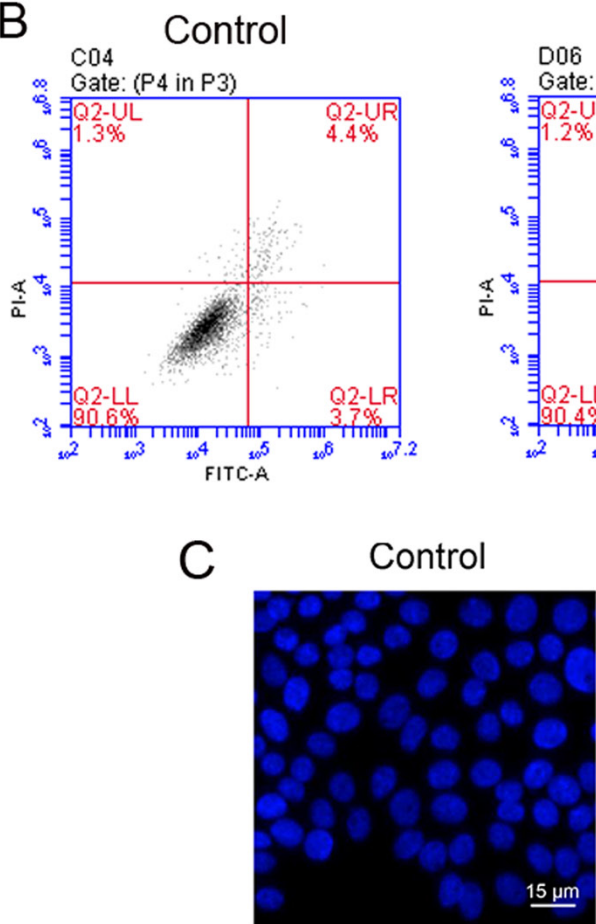

NC

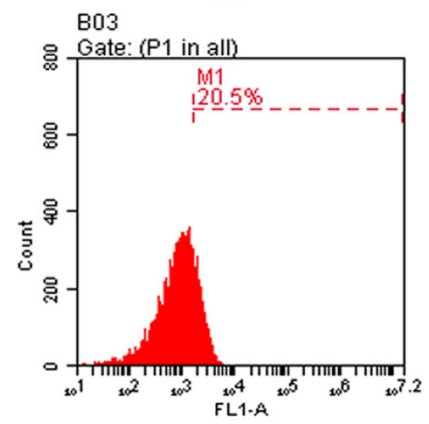

NC

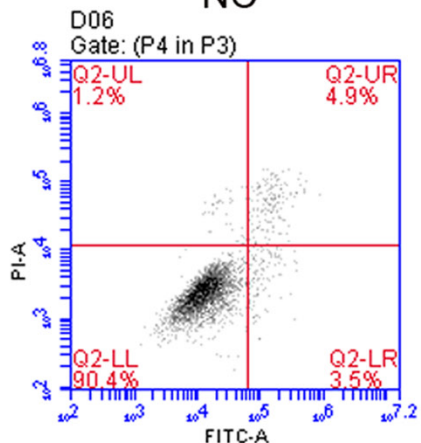

PF

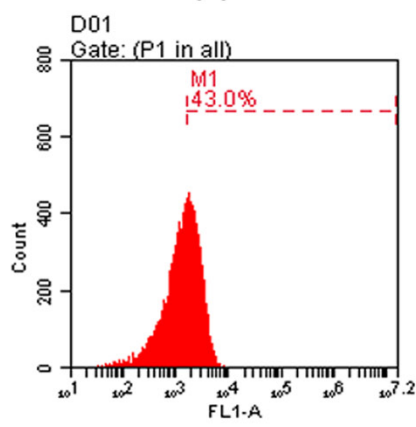

PF

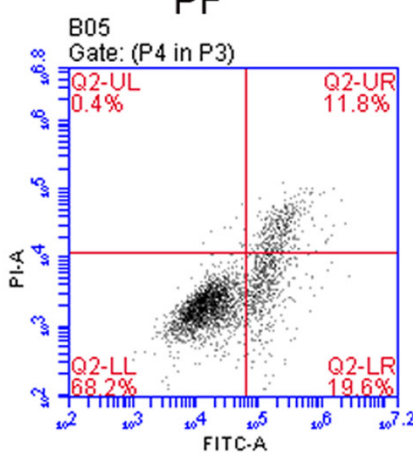

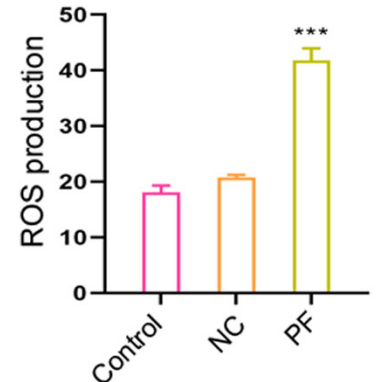

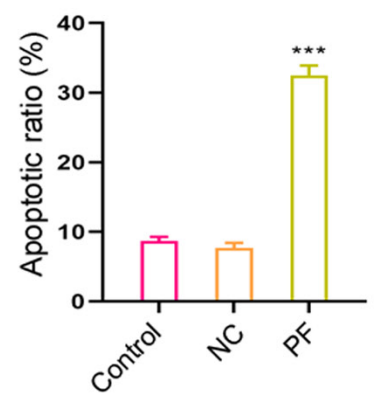

Figure 3. Determination of intracellular ROS levels and apoptotic hSGCs. (A) Intracellular ROS levels and (B) percentage of apoptotic hSGCs were determined by flow cytometry. (C) Hoechst 33258 staining of apoptotic cells. ${ }^{* * * *} \mathrm{P}<0.001$ vs. control PF, paeoniflorin. NC, negative control; ROS, reactive oxygen species; hSGC, human sweat gland cell.

significantly increased ROS production (Fig. 3A; $15.62 \pm 1.52$ vs. $39.44 \pm 2.42 \%$ ) and significantly promoted
hSGC apoptosis (Fig. 3B and C). PF treatment for $24 \mathrm{~h}$ increased the percentage of apoptotic hSGCs from 

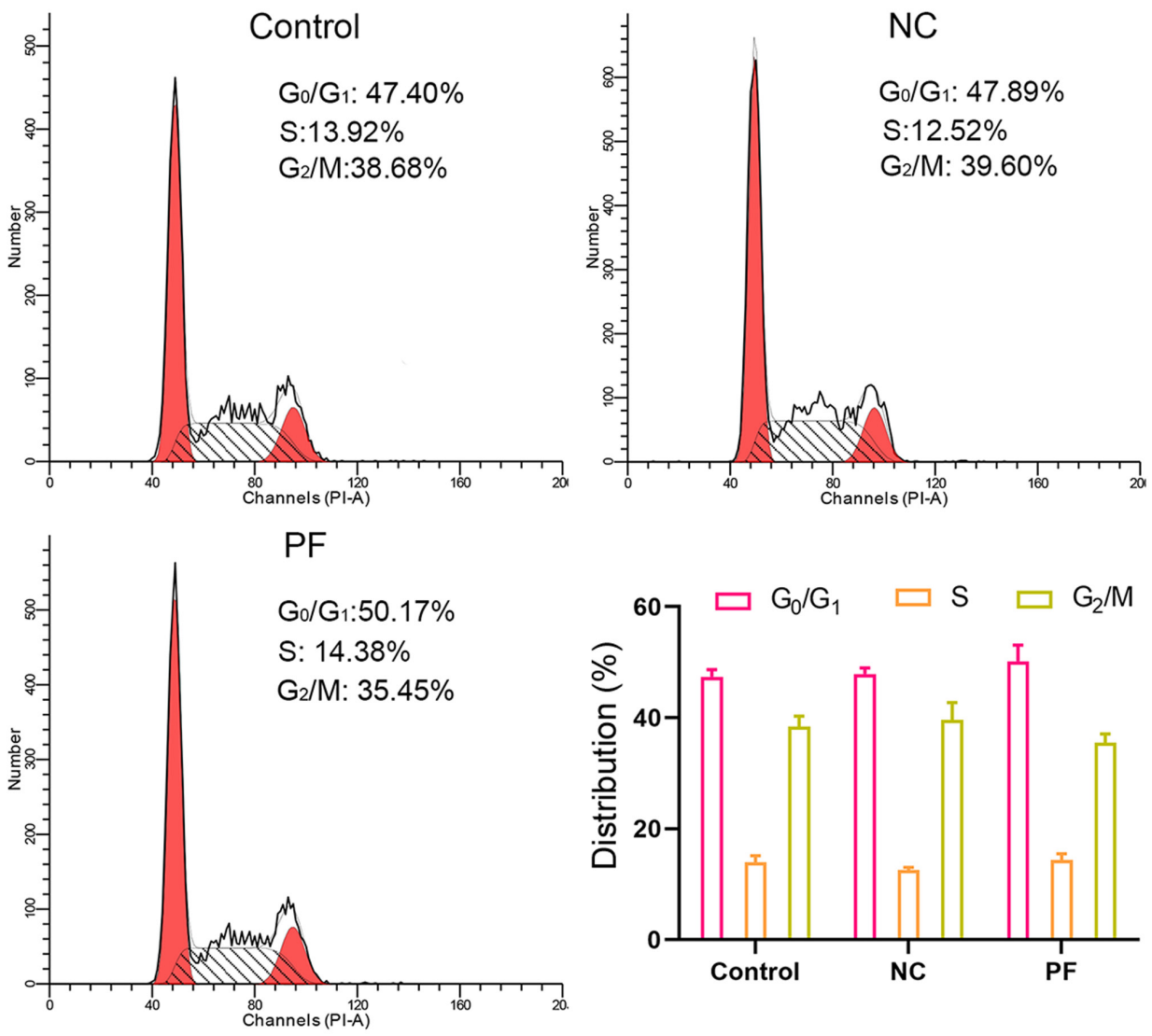

Figure 4. Cell cycle distribution of human sweat gland cells. Cell cycle distribution was analyzed by flow cytometry. PF, paeoniflorin. NC, negative control.

$6.35 \pm 0.67$ to $32.01 \pm 2.78 \%$. These results suggested PF exerted a cytotoxic effect on hSGCs.

PF alters cell cycle distribution. Flow cytometric analysis of cell cycle distribution showed that PF treatment for $24 \mathrm{~h}$ did not affect the cell cycle distribution of hSGCs. The percentage of hSGCs at G0/G1 phase following PF treatment was $59.05 \pm 5.91$ vs. $56.95 \pm 1.54 \%$ for control hSGCs (Fig. 4).

3-MA inhibits PF-induced ROS production and apoptosis in hSGCs Previous reports have demonstrated that PF promotes autophagy $(26,27)$. Here, 3 -MA, an autophagy inhibitor, partially suppressed ROS production and apoptosis in hSGCs that had been treated with PF (Fig. 5A and B); these results were confirmed by Hoechst 33258 staining (Fig. 5C). These findings showed that $\mathrm{PF}$-induced ROS production and apoptosis in hSGCs may be associated with promotion of autophagy.

Expression of autophagy-associated proteins following $P F$ and 3-MA treatment. To verify whether autophagy was associated with the molecular mechanism underlying PF-induced cellular changes in hSGCs, expression levels of autophagy-associated proteins were detected. PF alone significantly upregulated the levels of LC3B and Beclin 1 expression, but decreased p62 expression in hSGCs (Fig. 6A and B). By contrast, administration of autophagy inhibitor (3-MA) partially reversed the
PF-induced changes in LC3B, Beclin 1 and p62 expression (Fig. 6B). In addition, PF-induced inhibition of p-PI3K and p-Akt expression was also partially reversed by 3-MA (Fig. 6B). These protein expression profiles indicated the involvement of autophagy in PF-induced hSGC cytotoxicity.

\section{Discussion}

To the best of our knowledge, the present study is the first to report the inhibitory effect of PF on SGC proliferation. The in vivo results showed that PF inhibited SGC proliferation, while in vitro experiments showed that PF suppressed SGC proliferation and promoted apoptosis, autophagy and ROS production.

Cao et al (13) and Sun et al (28) showed that PF administration attenuates 1-methyl-4-phenylpyridinium-induced production of cytosolic free $\mathrm{Ca}^{2+}$ in PC12 cells and that levels of LC3-II protein are upregulated during this process $(13,28)$. Chen et al (29) reported that pretreatment with PF restored advanced glycation end product -modified bovine serum albumin-induced decreases in cell viability and p62 expression, but enhanced the expression of LC3-II in human umbilical vein endothelial cells (29). These studies suggested that PF promotes autophagy in cells.

Additionally, PF is reported to inhibit proliferation of different types of cell, including fibroblast-like synoviocytes (17), pulmonary artery (18) and vascular SMCs (30) and several types of tumor cell (31) by regulating various pathways, 
A

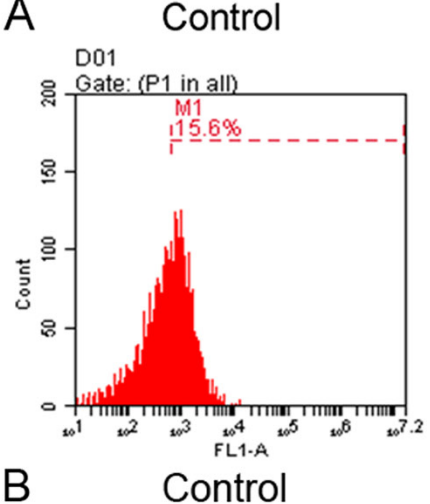

B

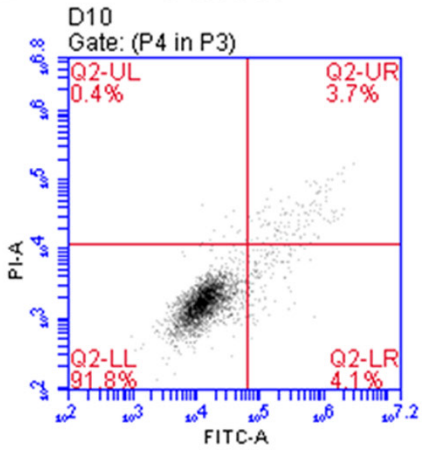

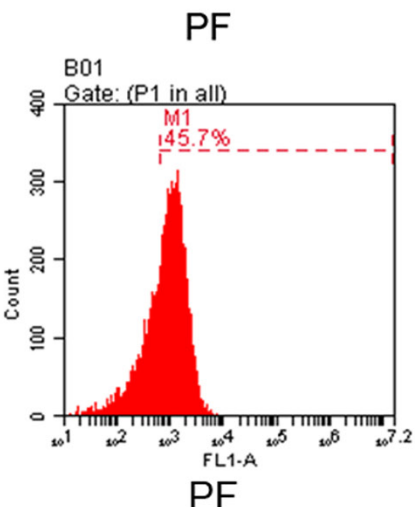

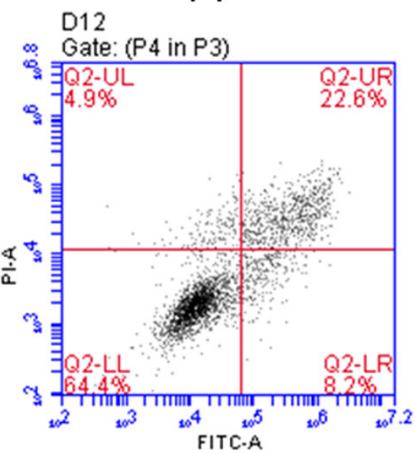

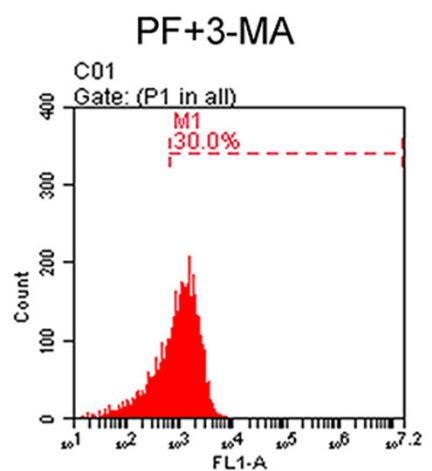

$\mathrm{PF}+3-\mathrm{MA}$

C11

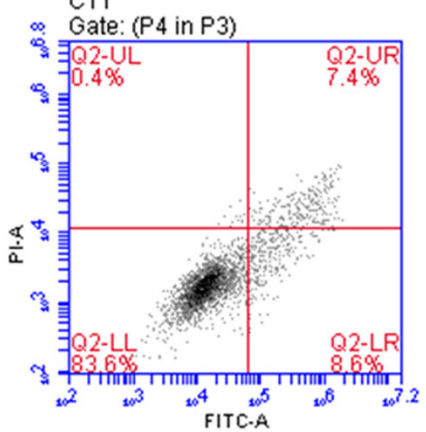

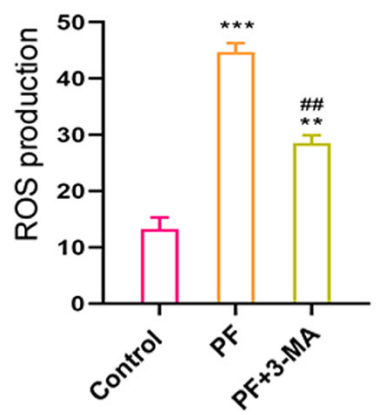

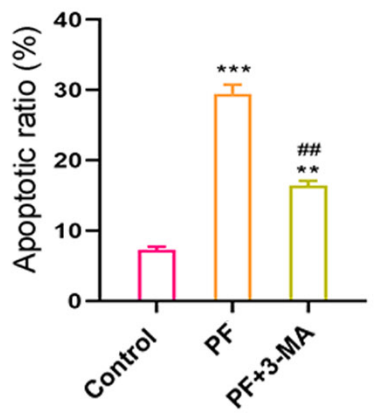

C

Control
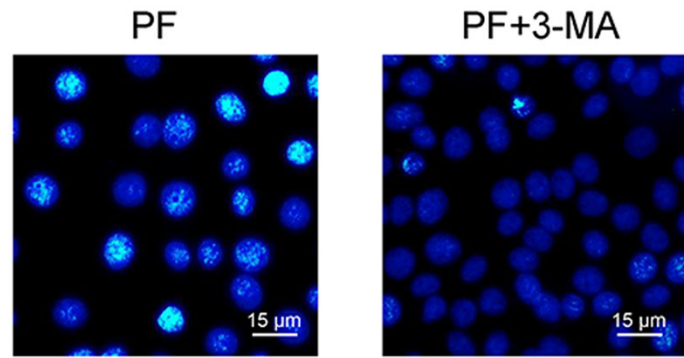

Figure 5.3-MA inhibits ROS production and apoptosis of hSGCs. (A) Intracellular ROS levels and (B) percentage of apoptotic hSGCs were determined by flow cytometry. (C) Hoechst 33258 staining of apoptotic cells. ${ }^{* *} \mathrm{P}<0.01,{ }^{* * *} \mathrm{P}<0.001$ vs. control; ${ }^{\# \#} \mathrm{P}<0.01$ vs. PF. PF, paeoniflorin; NC, negative control; ROS, reactive oxygen species; hSGC, human sweat gland cell; 3-MA, 3-methyladenine.

including the NF- $\mathrm{BB}$ pathway. In accordance with these findings, the present study showed that PF promoted autophagy and apoptosis in hSGCs cells by increasing LC3B and Beclin 1 expression and downregulating p62 expression. The PF-induced inhibition of hSGC proliferation was consistent with nuclear pyknosis observed in SGs of rat foot skin in vivo. In addition, PF-induced inhibition of hSGC proliferation was associated with downregulation of the pro-proliferative PI3K/Akt pathway. Accordingly, the present results showed that PF inhibited proliferation of hSGCs, potentially by inducing autophagy.

Zhao et al (32) showed that PF attenuates $\alpha$-naphthyl isothicaynate-induced ROS production in rats. In certain types of cell, activation of the PI3K/Akt pathway is ROS-dependent (33-35). However, the overproduction of ROS can damage cellular DNA and decrease cell membrane stability $(36,37)$. The interaction between ROS release and autophagy is complex and multifarious (38). ROS-induced autophagy and its mechanisms are commonly studied in cancer cells (38-40). In salivary gland cells, PF promotes production of intracellular $\mathrm{Ca}^{2+}(11)$; this effect can be triggered by ROS production, which trigger short-term autophagy in cells (41). The present study showed that ROS production promoted by PF in hSGCs was associated with apoptosis and autophagy; all these factors were altered by inhibition of autophagy. These findings suggest a complex mechanism by which PF inhibits hSGC proliferation. In summary, PF treatment suppressed SGC proliferation and promoted apoptosis, autophagy and ROS production, suggesting that PF may be useful for managing bromhidrosis. However, the exact mechanism by which PF reduces bromhidrosis was not fully clarifiedand requires further investigation.

To the best of our knowledge, the present study is the first to demonstrate that PF inhibits hSGC proliferation by promoting autophagy, ROS production and apoptosis. The in vivo anti-proliferative effect of PF on hSGCs was also confirmed. The present data revealed the effects of autophagy, ROS production, cell proliferation and apoptosis in the management of bromhidrosis by PF.

\section{Acknowledgements}

Not applicable. 

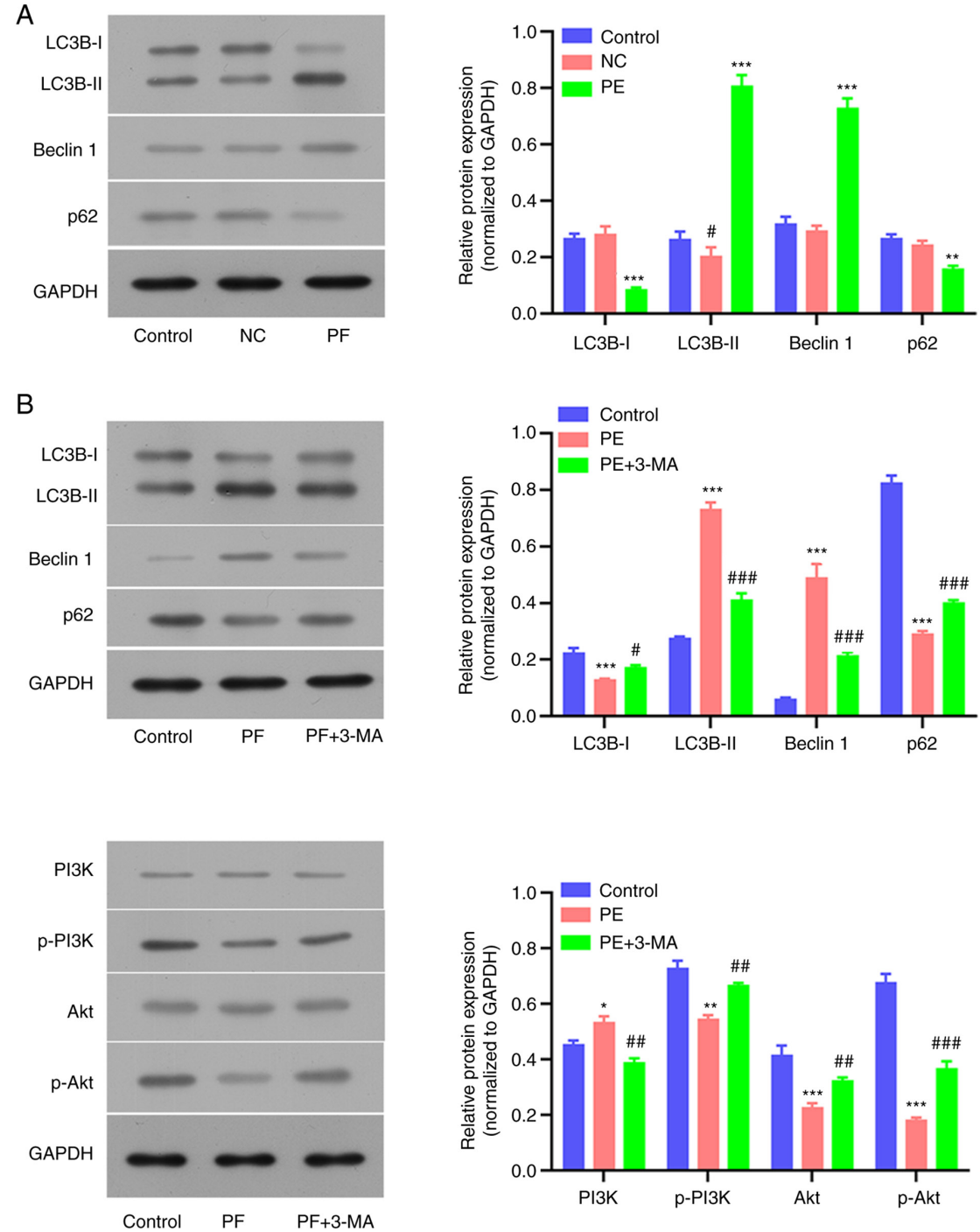

Figure 6. Expression of autophagy-associated proteins. (A) hSGCs were treated with $\mathrm{PF}$ (B) in the presence or absence of autophagy inhibitor 3-MA. "P<0.05, ${ }^{* *} \mathrm{P}<0.01,{ }^{* * *} \mathrm{P}<0.001$ vs. control; ${ }^{*} \mathrm{P}<0.05,{ }^{\# \#} \mathrm{P}<0.01,{ }^{\# \# \#} \mathrm{P}<0.001$ vs. PF. PF, paeoniflorin; NC, negative control; hSGC, human sweat gland cell; 3-MA, 3-methyladenine; p-, phosphorylated.

\section{Funding}

No funding was received.

\section{Availability of data and materials}

The datasets generated and/or analyzed during the present study are available from the corresponding author on reasonable request.

\section{Authors' contributions}

YX, HH and PL conceived, designed and performed the experiments. HH, PL and HL analyzed and interpreted data. YX and HL wrote and revised the manuscript. YX and HL confirm the authenticity of all the raw data. All authors have read and approved the final version of the manuscript.

\section{Ethics approval and consent to participate}

The protocols for all animal experiments were approved by the Institutional Animal Care and Use Committee of the First
Affiliated Hospital of Guangdong Pharmaceutical University (approval no. 202182). All patients provided written informed consent. The protocol for the clinical study was approved by the Research Ethics Committee of the First Affiliated Hospital of Guangdong Pharmaceutical University (approval no. gyfykydw039). All procedures were performed in compliance with Ethics Committee regulations and Animal Research: Reporting of In Vivo Experiments guidelines.

\section{Patient consent for publication}

Not applicable.

\section{Competing interests}

The authors declare have no competing interests.

\section{References}

1. Hsu KC and Wang KY: Sparing subcutaneous septa avoids skin necrosis in the treatment of axillary bromhidrosis with suction-curettage shaving. J Cosmet Dermatol 18: 892-896, 2019. 
2. Ardon CB, Molenaar C, van Straalen KR, Scholtes VC, Prens EP and van der Zee HH: High prevalence of hidradenitis suppurativa in patients with perianal fistula. Int J Colorectal Dis 34: 1337-1339, 2019.

3. Van TN, Manh TN, Minh PPT, Minh TT, Huu ND, Cao KP, Huu QN, Cam VT, Huyen ML, Hau KT, et al: The Effectiveness of Local Surgical Technique in Treatment of Axillary Bromhidrosis. Open Access Maced J Med Sci 7: 187-191, 2019.

4. He J, Wang T and Dong J: A close positive correlation between malodor and sweating as a marker for the treatment of axillary bromhidrosis with Botulinum toxin A. J Dermatolog Treat 23: 461-464, 2012

5. Kataoka A: Surgical treatment of bromhidrosis. Rev Bras Cir Plást 32: 377-382, 2001.

6. Coronado MS and Opi JT: Assessment of axillary hyperhidrosis and bromhidrosis treatment with microwave technology. J Surg Med 3: 447-451, 2019.

7. Mao GY, Yang SL and Zheng JH: Etiology and management of axillary bromidrosis: A brief review. Int $\mathrm{J}$ Dermatol 47: 1063-1068, 2008.

8. Mancini M, Panasiti V, Devirgiliis V, Pietropaolo V, Fioriti D, Nicosia R, Curzio M, Roberti V, Gobbi S, Bottoni $\mathrm{U}$, et al: Bromhidrosis induced by sphingomonas paucimobilis: A case report. Int J Immunopathol Pharmacol 22: 845-848, 2009.

9. Hashmonai M, Cameron AEP, Connery CP, Perin $\mathrm{N}$ and Licht PB: The etiology of primary hyperhidrosis: A systematic review. Clin Auton Res 27: 379-383, 2017.

10. Zhang Y, Hu S, Ge S, Wang J and He L: Paeoniflorin inhibits IgE-mediated allergic reactions by suppressing the degranulation of mast cells though binding with FceRI alpha subunits. Eur J Pharmacol 886: 173415, 2020.

11. Qian X, Shi X and Wang H: Effect of paeoniflorin on the calcium ion concentration in salivary gland cells using confocal laser scanning microscopy. Am J Transl Res 8: 3678-3688, 2016.

12. Manayi A, Omidpanah S, Barreca D, Ficarra S, Daglia M, Nabavi SF and Nabavi SM: Neuroprotective effects of paeoniflorin in neurodegenerative diseases of the central nervous system. Phytochem Rev 16: 1173-1181, 2017.

13. Cao B-Y, Yang Y-P, Luo W-F, Mao CJ, Han R, Sun X, Cheng J and Liu CF: Paeoniflorin, a potent natural compound, protects PC12 cells from MPP+ and acidic damage via autophagic pathway. J Ethnopharmacol 131: 122-129, 2010.

14. Wu X, Sun X, Zhao C, Zhang J, Wang X, Zhang A and Wang X: Exploring the pharmacological effects and potential targets of paeoniflorin on the endometriosis of cold coagulation and blood stasis model rats by ultra-performance liquid chromatography tandem mass spectrometry with a pattern recognition approach. RSC Advances 9: 20796-20805, 2019.

15. Hu H, Zhu Q, Su J, Wu Y, Zhu Y, Wang Y, Fang H, Pang M, $\mathrm{Li} \mathrm{B}$, Chen S, et al: Effects of an enriched extract of paeoniflorin, a monoterpene glycoside used in Chinese herbal medicine, on cholesterol metabolism in a hyperlipidemic rat model. Med Sci Monit 23: 3412-3427, 2017

16. Zhang L, Yang B and Yu B: Paeoniflorin protects against nonalcoholic fatty liver disease induced by a high-fat diet in mice. Biol Pharm Bull 38: 1005-1011, 2015.

17. Chen J-Y, Wu H-X, Chen Y, Zhang LL, Wang QT, Sun WY and Wei W: Paeoniflorin inhibits proliferation of fibroblast-like synoviocytes through suppressing G-protein-coupled receptor kinase 2. Planta Med 78: 665-671, 2012.

18. Qian G, Cao J, Chen C, Wang L, Huang X, Ding C, Cai X, Yin F, Chu J, Li G, et al: Paeoniflorin inhibits pulmonary artery smooth muscle cells proliferation via upregulating A2B adenosine receptor in rat. PLoS One 8: e69141, 2013.

19. Ferry B, Gervasoni D and Vogt C: Regulatory and Ethical Considerations. In: Stereotaxic Neurosurgery in Laboratory Rodent. Springer, Paris, pp1-18, 2014

20. Turkki R, Linder N, Kovanen PE, Pellinen T and Lundin J: Identification of immune cell infiltration in hematoxylin-eosin stained breast cancer samples: texture-based classification of tissue morphologies. In: Proceedings of SPIE - The International Society for Optical Engineering 9791. Medical Imaging 2016: Digital Pathology, San Diego, p9791, 2016.

21. Park Y-J and Shin M-S: What is the best method for treating osmidrosis? Ann Plast Surg 47: 303-309, 2001

22. Huang Z, Zhen Y, Yin W, Ma Z and Zhang L: Shh promotes sweat gland cell maturation in three-dimensional culture. Cell Tissue Bank 17: 317-325, 2016.
23. Kolakshyapati P,Li X, Chen C, Zhang M, Tan W, Ma L and Gao C Gene-activated matrix/bone marrow-derived mesenchymal stem cells constructs regenerate sweat glands-like structure in vivo. Sci Rep 7: 17630, 2017.

24. Kurata R, Futaki S, Nakano I, Fujita F, Tanemura A, Murota H Katayama I, Okada F and Sekiguchi K: Three-dimensional cell shapes and arrangements in human sweat glands as revealed by whole-mount immunostaining. PLoS One 12: e0178709, 2017.

25. Huang J, Qiu L, Ding L, Wang S, Wang J, Zhu Q, Song F and Hu J: Ginsenoside Rb1 and paeoniflorin inhibit transient receptor potential vanilloid-1-activated IL-8 and PGE $_{2}$ production in a human keratinocyte cell line HaCaT. Int Immunopharmacol 10: 1279-1283, 2010.

26. Wen J, Xu B, Sun Y, Lian M, Li Y, Lin Y, Chen D, Diao Y, Almoiliqy $\mathrm{M}$ and Wang L: Paeoniflorin protects against intestinal ischemia/reperfusion by activating LKB1/AMPK and promoting autophagy. Pharmacol Res 146: 104308, 2019.

27. Wang Y, Che J, Zhao H, Tang J and Shi G: Paeoniflorin attenuates oxidized low-density lipoprotein-induced apoptosis and adhesion molecule expression by autophagy enhancement in human umbilical vein endothelial cells. J Cell Biochem 120: 9291-9299, 2019.

28. Sun X, Cao Y-B, Hu L-F, Yang YP, Li J, Wang F and Liu CF: ASICs mediate the modulatory effect by paeoniflorin on $\alpha$-synuclein autophagic degradation. Brain Res 1396: 77-87, 2011.

29. Chen Y, Du X, Zhou Y, Zhang Y, Yang Y, Liu Z, Liu C and Xie Y: Paeoniflorin protects HUVECs from AGE-BSA-induced injury via an autophagic pathway by acting on the RAGE. Int J Clin Exp Pathol 8: 53-62, 2015.

30. Li W, Zhi W, Liu F, Zhao J, Yao Q and Niu X: Paeoniflorin inhibits VSMCs proliferation and migration by arresting cell cycle and activating $\mathrm{HO}-1$ through MAPKs and NF- $\kappa$ B pathway. Int Immunopharmacol 54: 103-111, 2018.

31. Zheng Y-B, Xiao G-C, Tong S-L, Ding Y, Wang QS, Li SB and Hao ZN: Paeoniflorin inhibits human gastric carcinoma cell proliferation through up-regulation of microRNA-124 and suppression of PI3K/Akt and STAT3 signaling. World J Gastroenterol 21: 7197-7207, 2015.

32. Zhao Y, Zhou G, Wang J, Jia L, Zhang P, Li R, Shan L, Liu B, Song X, Liu S, et al: Paeoniflorin protects against ANIT-induced cholestasis by ameliorating oxidative stress in rats. Food Chem Toxicol 58: 242-248, 2013.

33. Wagner G, Lindroos-Christensen J, Einwallner E, Husa J, Zapf TC, Lipp K, Rauscher S, Gröger M, Spittler A, Loewe R, et al: HO-1 inhibits preadipocyte proliferation and differentiation at the onset of obesity via ROS dependent activation of Akt2. Sci Rep 7: 40881, 2017.

34. Mittler R: ROS are good. Trends Plant Sci 22: 11-19, 2017.

35. Wang X, Liu JZ, Hu JX, Wu H, Li YL, Chen HL, Bai H and Hai CX: ROS-activated p38 MAPK/ERK-Akt cascade plays a central role in palmitic acid-stimulated hepatocyte proliferation. Free Radic Biol Med 51: 539-551, 2011.

36. Ruiz-Ramos R, Lopez-Carrillo L, Rios-Perez AD, De Vizcaya-Ruíz A and Cebrian ME: Sodium arsenite induces ROS generation, DNA oxidative damage, HO-1 and c-Myc proteins, NF-kappaB activation and cell proliferation in human breast cancer MCF-7 cells. Mutat Res 674: 109-115, 2009.

37. Kadowaki H, Nishitoh H, Urano F, Sadamitsu C, Matsuzawa A, Takeda K, Masutani H, Yodoi J, Urano Y, Nagano T, et al: Amyloid $\beta$ induces neuronal cell death through ROS-mediated ASK1 activation. Cell Death Differ 12: 19-24, 2005.

38. Poillet-Perez L, Despouy G, Delage-Mourroux R and Boyer-Guittaut M: Interplay between ROS and autophagy in cancer cells, from tumor initiation to cancer therapy. Redox Biol 4: 184-192, 2015.

39. Li HY, Zhang J, Sun LL, Li BH, Gao HL, Xie T, Zhang N and Ye ZM: Celastrol induces apoptosis and autophagy via the ROS/JNK signaling pathway in human osteosarcoma cells: An in vitro and in vivo study. Cell Death Dis 6: e1604, 2015.

40. Liu D, Lin J, Su J, Chen X, Jiang P and Huang K: Glutamine deficiency promotes PCV2 infection through induction of autophagy via activation of ROS-mediated JAK2/STAT3 signaling pathway. J Agric Food Chem 66: 11757-11766, 2018.

41. Borodkina AV, Shatrova AN, Deryabin PI, Griukova AA, Abushik PA, Antonov SM, Nikolsky NN and Burova EB: Calcium alterations signal either to senescence or to autophagy induction in stem cells upon oxidative stress. Aging (Albany NY) 8: 3400-3418, 2016.

This work is licensed under a Creative Commons Attribution-NonCommercial-NoDerivatives 4.0 International (CC BY-NC-ND 4.0) License. 